\title{
Pacific
}

Journal of

Mathematics

\section{THE DIRICHLET PROBLEM \\ FOR HARMONIC FUNCTIONS ON COMPACT SETS}

TONY L. PERKINS 


\title{
THE DIRICHLET PROBLEM FOR HARMONIC FUNCTIONS ON COMPACT SETS
}

\author{
TONY L. PERKINS
}

\begin{abstract}
The main goal of this paper is to study the Dirichlet problem on a compact set $K \subset \mathbb{R}^{n}$. Initially we consider the space $H(K)$ of functions on $K$ that can be uniformly approximated by functions harmonic in a neighborhood of $K$ as possible solutions. As in the classical theory, we show $C\left(\partial_{f} K\right) \cong H(K)$ for compact sets with $\partial_{f} K$ closed, where $\partial_{f} K$ is the fine boundary of $K$. However, in general, a continuous solution cannot be expected, even for continuous data on $\partial_{f} K$. Consequently, we show that for any bounded continuous boundary data on $\partial_{f} K$, the solution can be found in a class of finely harmonic functions. Also, in complete analogy with the classical situation, this class is isometrically isomorphic to the set of bounded continuous functions on $\partial_{f} K$ for all compact sets $K$.
\end{abstract}

\section{Introduction}

The Dirichlet problem for harmonic functions on domains in $\mathbb{R}^{n}$ is important not only for its own sake but also because of its influence on potential theory. Many now-standard notions - regular points, fine topology, etc. - first appeared in the study of this problem. The main goal of this paper is to extend the classic theory to compact sets $K \subset \mathbb{R}^{n}$.

One possible extension can be found in the abstract theory of balayage spaces [Bliedtner and Hansen 1986; Hansen 1985]. However, we feel that the gain in transparency resulting from a direct geometric approach more than justifies the use of new techniques.

The Dirichlet problem can be thought of as having two components: the data set and the data itself. One uses an initial function defined on the data set to construct a solution (a harmonic function) on the rest of the domain, which must have a prescribed regularity as it approaches the data set. Classically, the data set is taken to be the topological boundary of the domain. One of the main goals of this paper is to establish that the natural choice for the data set on compact sets is the fine

MSC2010: primary 31B05; secondary 31B10, 31B25, 31C40.

Keywords: Harmonic measure, Jensen measures, subharmonic functions, potential theory, fine topology. 
boundary $\partial_{f} K$ of $K$, which is shown in Lemma 5.1 to be the Choquet boundary of $K$ with respect to subharmonic functions on $K$. We limit ourselves to initial functions that are continuous and bounded on $\partial_{f} K$, as in the classical case.

In Section 3, we introduce Jensen measures as our main tool and extend potential theory to compact sets $K \subset \mathbb{R}^{n}$ by defining harmonic functions and subharmonic functions on $K$. We devote Section 4 to the construction and study of harmonic measures on compact sets. The harmonic measure on $K$ is shown to be a maximal Jensen measure. This is used to see that harmonic measures are concentrated on the fine boundary (Corollary 5.3). In Section 6 we study the Dirichlet problem for compact sets. As in the classical theory, our Theorem 6.1 shows $C\left(\partial_{f} K\right) \cong H(K)$ for a class of compact sets. However, in general, a continuous solution cannot be expected, even for continuous data on $\partial_{f} K$, as we illustrate in Example 6.2. Therefore we show that the solution can be found in the class of finely harmonic functions introduced in that section. By Theorem 6.5, in complete analogy with the classical situation, this class is isometrically isomorphic to the set of bounded continuous functions on $\partial_{f} K$, denoted $C_{b}\left(\partial_{f} K\right)$, for all compact sets $K$.

\section{Basic facts}

Let $M(\Omega)$ denote the space of finite signed Radon measures on $\Omega \subset \mathbb{R}^{n}$, and $C_{0}\left(\mathbb{R}^{n}\right)$ the space of continuous functions on $\mathbb{R}^{n}$ that vanish at infinity. We often use $\mu(f)$ to denote $\int f d \mu$.

Classical potential theory. Let $D$ be an open set in $\mathbb{R}^{n}$, with $n \geq 2$. For any $f \in C(\partial D)$, the Dirichlet problem on $D$ is to find a unique function $h$ that is harmonic on $D$ and continuous on $\bar{D}$ such that $\left.h\right|_{\partial D}=f$. The function $f$ is commonly referred to as the boundary data, and the corresponding $h$ is said to be the solution of the Dirichlet problem on $D$ with boundary data $f$. The punctured disk in $\mathbb{R}^{2}$ is a fundamental example that shows that the Dirichlet problem cannot be solved for any continuous boundary data. However, for a bounded open set $U$, the method of Perron allows one to assign a function that is harmonic on $U$ to any continuous (or simply measurable) boundary data. The concept of a regular domain was developed to establish the continuity of the Perron solution to the boundary. A bounded connected open set $D \subset \mathbb{R}^{n}$ is a regular domain if the Dirichlet problem is solvable on $D$ for any continuous boundary data. Therefore, on a regular domain, $C(\partial D)$ is isometrically isomorphic to $H(D)$, the space of continuous functions on $\bar{D}$ that are harmonic on $D$. For any $f \in C(\partial D)$, let $h_{f} \in H(D)$ denote the solution of the Dirichlet problem on $D$ with boundary data $f$. Put $z \in D$. The point evaluation

$$
H_{z}: f \mapsto h_{f}(z)
$$


is a positive bounded linear functional on $C(\partial D)$. By the Riesz representation theorem, there is a Radon measure $\omega_{D}(z, \cdot)$ on $\partial D$ that represents $H_{z}$; that is,

$$
h_{f}(z)=\int_{\partial D} f(\zeta) d \omega_{D}(z, \zeta)
$$

for all $f \in C(\partial D)$. The measure $\omega_{D}(z, \cdot)$ is called the harmonic measure of $D$ with barycenter at $z$. See [Armitage and Gardiner 2001] for more details on potential theory.

Jensen measures. If $D$ is an open set in $\mathbb{R}^{n}$, we call $\mu$ a Jensen measure on $D$ with barycenter $z \in D$ if $\mu$ is a probability measure (a positive Radon measure of unit mass) whose support is compactly contained in $D$ and if for every subharmonic function $f$ on $D$ the subaveraging inequality $f(z) \leq \mu(f)$ holds. The set of Jensen measures on $D$ with barycenter $z \in D$ we denote by $\mathscr{F}_{z}(D)$.

One could define the set of Jensen measures $\mathscr{g}_{z}^{c}(D)$ with respect to the continuous subharmonic functions on $D$. However, the following theorem shows that the set of Jensen measures would not be changed.

Theorem 2.1. Let $D$ be a bounded open subset of $\mathbb{R}^{n}$. For every $z \in D$, the sets $\mathscr{F}_{z}(D)$ and $\mathscr{F}_{z}^{c}(D)$ are equal.

Proof. Since clearly $\mathscr{g}_{z}(D) \subseteq \mathscr{g}_{z}^{c}(D)$ for all $z \in D$, we show the reverse inclusion.

Pick some $z_{0} \in D$ and let $\mu \in \mathscr{F}_{z_{0}}^{c}(D)$. Then we must show $f\left(z_{0}\right) \leq \mu(f)$ for every function $f$ that is subharmonic on $D$. The support of $\mu$ is compactly contained in $D$.

Because $f$ is subharmonic on $D$, we can find a decreasing sequence $\left\{f_{n}\right\}$ of continuous subharmonic functions that converge to $f$. Since $\mu \in \mathscr{F}_{z_{0}}^{c}(D)$, we have $f\left(z_{0}\right) \leq \mu\left(f_{n}\right)$ for every $f_{n}$. By the Lebesgue monotone convergence theorem, it follows that $f\left(z_{0}\right) \leq \mu(f)$. Thus $\mu \in \mathscr{F}_{z_{0}}(D)$.

Since $\mathscr{g}_{z}(D)=\mathscr{F}_{z}^{c}(D)$ for all $z \in D$, to check that $\mu \in \mathscr{F}_{z}(D)$ it suffices to check that $\mu$ has the subaveraging property for every continuous subharmonic function.

Examples of Jensen measures with barycenter at $z \in D$ include the Dirac measure at $z$, that is, $\delta_{z}$, the harmonic measure with barycenter at $z$ for any regular domain that is compactly contained in $D$, and the average over any ball (or sphere) centered at $z$ that is contained in $D$. The following proposition demonstrates some basic properties of sets of Jensen measures.

Proposition 2.2 [Cole and Ransford 2001, Proposition 2.1]. Let $D_{1}$ and $D_{2}$ be open subsets of $\mathbb{R}^{n}$ with $D_{1} \subset D_{2}$. Let $z \in D_{1}$.

(i) If $\mu \in \mathscr{F}_{z}\left(D_{1}\right)$, then also $\mu \in \mathscr{F}_{z}\left(D_{2}\right)$.

(ii) If $\mu \in \mathscr{F}_{z}\left(D_{2}\right)$ and $\operatorname{supp}(\mu) \subset D_{1}$, and if each bounded component of $\mathbb{R}^{n} \backslash D_{1}$ meets $\mathbb{R}^{n} \backslash D_{2}$, then $\mu \in \mathscr{g}_{z}\left(D_{2}\right)$. 
Jensen measures and subharmonic functions are, in a sense, dual to each other. This duality is illustrated by the following theorem.

Theorem 2.3 [Cole and Ransford 1997, Corollary 1.7]. Let D be an open subset of $\mathbb{R}^{n}$ that possesses a Green's function. Let

$$
\phi: D \rightarrow[-\infty, \infty)
$$

be a Borel measurable function that is locally bounded above. Then

$$
\sup \{v(z): v \in S(D), v \leq \phi\}=\inf \left\{\mu(\phi): \mu \in \mathscr{F}_{z}(D)\right\}
$$

for each $z \in D$, where $S(D)$ denotes the set of subharmonic functions on $D$.

Fine topology. The two books [Brelot 1971; Fuglede 1972] are classical references on the fine topology, and many books on potential theory contain chapters on the topic, for example [Armitage and Gardiner 2001, Chapter 7].

The fine topology on $\mathbb{R}^{n}$ is the coarsest topology on $\mathbb{R}^{n}$ such that all subharmonic functions are continuous in the extended sense of taking values in $[-\infty, \infty]$.

When referring to a topological concept in the fine topology, we follow the standard policy of either using the words "fine" or "finely" prior to the topological concept, or attaching the letter $f$ to the associated symbol. For example, the fine boundary of $K, \partial_{f} K$, is the boundary of $K$ in the fine topology. The fine topology is strictly finer than the Euclidean topology.

Many of the key concepts of classical potential theory have analogous definitions in relation to the fine topology. We recall a few of them. Relative to a finely open set $V$ in $\mathbb{R}^{n}$, the harmonic measure $\delta_{x}^{V^{c}}$ is defined as the swept-out of the Dirac measure $\delta_{x}$ on the complement of $V$. A function $u$ is said to be finely hypoharmonic on a finely open set $U$ if it is upper finite, finely upper semicontinuous, and if

$$
u(x) \leq \delta_{x}^{V^{c}}(u)<\infty
$$

for all $x \in V$ and all relatively compact finely open sets $V$ with fine closure contained in $U$. A function $h$ is said to be finely harmonic if $h$ and $-h$ are finely hypoharmonic. Also, the fine Dirichlet problem on $U$ for a finely continuous function $f$ defined on the fine boundary of a bounded finely open set $U$ consists of finding a finely harmonic extension of $f$ to $U$. The development of the fine Dirichlet problem is quite similar to that of the classical. Fuglede [1972, Theorem 14.6] establishes a Perron solution for the fine Dirichlet problem, showing that there exists a Perron solution $H_{f}^{U}$ that is finely harmonic on $U$ for any numerical function $f$ on $\partial_{f} U$ that is $\delta_{x}^{\partial_{f} U}$ integrable for every $x \in U$. The same theorem also provides us with the desired continuity at the boundary, showing that the fine limit of $H_{f}^{U}(x)$ tends to $f(y)$ as $x \in U$ goes to $y$ for every finely "regular" boundary point $y \in \partial_{f} U$ at which $f$ is finely continuous. 


\section{Harmonic and subharmonic functions on compact sets}

We now begin our study of potential theory on compact sets. For compact sets that are not connected, the Hausdorff property allows us to reduce Dirichlet-type problems on the compact set to solving separate problems on each connected component. Therefore, in what follows, we work on compact sets $K$ in $\mathbb{R}^{n}$ that need not be connected, with the understanding that we can always separate the problem by working on the connected components of $K$ individually.

There are currently three equivalent ways to define harmonic and subharmonic functions on compact sets.

Definition 3.1 (exterior). Let $H(K)$ (resp. $S(K)$ ) be the uniform closures of all functions in $C(K)$ that are restrictions of harmonic (resp. subharmonic) functions on a neighborhood of $K$.

Definition 3.2 (interior). One can define $H(K)$ and $S(K)$ as the subspaces of $C(K)$ consisting of functions that are finely harmonic and finely subharmonic, respectively, on the fine interior of $K$.

The equivalence of these definitions of $H(K)$ was shown in [Debiard and Gaveau 1974], and of $S(K)$ in [Bliedtner and Hansen 1975; 1978].

For the third definition of $H(K)$, we extend the notion of Jensen measures to compact sets.

Definition 3.3. We define the set of Jensen measures on $K$ with barycenter at $z \in K$ as the intersection of all sets $\mathscr{g}_{z}(U)$, that is,

$$
\mathscr{E}_{z}(K)=\bigcap_{K \subset U} \mathscr{F}_{z}(U),
$$

where $U$ is any open set containing $K$.

Another definition of $H(K)$ was introduced in [Poletsky 1997] using the notion of Jensen measures.

Definition 3.4 (via Jensen measures). The set $H(K)$ is the subspace of $C(K)$ consisting of functions $h$ such that $h(x)=\mu(h)$ for all $\mu \in \mathscr{F}_{x}(K)$ and $x \in K$.

It was shown in [Poletsky 1997] that this definition is equivalent to the exterior definition above.

The next lemma shows that this last construction extends to subharmonic functions in the ideal way.

Lemma 3.5. A function is in $S(K)$ if and only if it is continuous and satisfies the subaveraging property with respect to every Jensen measure on $K$; that is,

$$
S(K)=\left\{f \in C(K): f(z) \leq \mu(f), \text { for all } \mu \in \mathscr{F}_{z}(K) \text { and every } z \in K\right\} .
$$


Proof. We use the exterior definition of $S(K)$ to show "ᄃ". Take $f \in C(K)$ and let $\left\{f_{j}\right\}$ be a sequence of subharmonic functions defined in a neighborhood of $K$ such that $\left\{f_{j}\right\}$ is converging uniformly to $f$. Then $f_{j}(z) \leq \mu\left(f_{j}\right)$ for any $\mu \in \mathscr{F}_{z}(K)$. Because the convergence is uniform, we have $f(z) \leq \mu(f)$.

Now suppose that $f$ is in the set on the right. The subaveraging condition implies that $f$ is finely subharmonic on the fine interior of $K$, and by assumption, $f$ is continuous. Therefore $f$ satisfies the interior definition of $S(K)$.

Recall the exterior definition of $S(K)$ as the uniform limits of continuous functions subharmonic in neighborhoods of $K$. Proposition 3.6 shows that the defining sequence for any function in $S(K)$ may be taken to be increasing. This is a simple consequence of a duality theorem of Edwards [Gamelin 1978, Theorem 1.2; Cole and Ransford 1997].

Proposition 3.6. Every function in $S(K)$ is the limit of an increasing sequence of continuous subharmonic functions defined on neighborhoods of $K$.

Proof. Edwards's theorem states that if $p$ is a continuous function on $K$, then for all $z \in K$ we have

$$
E p(z):=\sup \{f(z): f \in S(K), f \leq p\}=\inf \left\{\mu(p): \mu \in \mathscr{F}_{z}(K)\right\} .
$$

From the proof of this theorem, it follows that Ep is lower semicontinuous and is the limit of an increasing sequence of continuous subharmonic functions on neighborhoods of $K$. The result follows by observing that $p=E p$ whenever $p \in S(K)$.

\section{Harmonic measure on a compact set}

To use the exterior definition of $H(K)$, we typically want to approximate $K$ by a decreasing sequence of regular domains. A decreasing sequence of regular domains $\left\{U_{j}\right\}$ is said to be converging to $K$ if for every $\varepsilon>0$, there is a $j_{0}$ such that $U_{j}$ contains $K$ and lies in the $\varepsilon$-neighborhood $K_{\varepsilon}$ of $K$ when $j \geq j_{0}$. Furthermore, we require that $U_{j+1}$ be compactly contained in $U_{j}$; that is, $\bar{U}_{j+1} \subset U_{j}$ for all $j$. The existence of such a sequence is provided by [Hervé 1962, Proposition 7.1].

Theorem 4.1 allows us to define a harmonic measure on $K$. For a decreasing sequence of regular domains $\left\{U_{j}\right\}$, we let $\omega_{U_{j}}(z, \cdot)$ denote the harmonic measure on $U_{j}$ with barycenter at $z \in U_{j}$.

Theorem 4.1. If $\left\{U_{j}\right\}$ is a sequence of regular domains converging to a compact set $K \subset \mathbb{R}^{n}$, then for every $z \in K$, the harmonic measures $\omega_{U_{j}}(z, \cdot)$ converge weak ${ }^{*}$. Also, this limit does not depend on the choice of the sequence of domains $\left\{U_{j}\right\}$.

Proof. Since $\omega_{U_{j}}$ are measures of unit mass supported on a compact set in $\mathbb{R}^{n}$, by Alaoglu's theorem they must have a limit point. To show that this point is unique, 
it suffices to show that for every $z \in K$, the limit

$$
\lim _{j \rightarrow \infty} \int_{\partial U_{j}} u(\zeta) d \omega_{U_{j}}(z, \zeta)
$$

exists for every $u \in C\left(\bar{U}_{1}\right)$.

First, we show that the limit in (1) exists when $u$ is continuous and subharmonic in a neighborhood of $K$. The solution $u_{j}$ of the Dirichlet problem on $U_{j}$ with boundary value $u$ is equal to

$$
u_{j}(z)=\int_{\partial U_{j}} u(\zeta) d \omega_{U_{j}}(z, \zeta)
$$

Since $u$ is subharmonic, we have $u_{j} \geq u$ on $U_{j}$. Then, since $u_{j+1}=u$ on $\partial U_{j+1}$ and $u_{j} \geq u=u_{j+1}$ on $\partial U_{j+1}$, the maximum principle for harmonic functions implies that $u_{j} \geq u_{j+1}$ on $U_{j+1}$. Thus $\left\{u_{j}\right\}$ is a decreasing sequence on $K$, and we see that for every $z \in K$, the limit in (1) exists.

If $u \in C^{2}\left(\bar{U}_{1}\right)$, then we may represent $u$ as a difference of two $C^{2}\left(\bar{U}_{1}\right)$ functions that are subharmonic on $U_{1}$. By the argument above, the limit in (1) exists.

Because $C^{2}\left(\bar{U}_{1}\right)$ is dense in $C\left(\bar{U}_{1}\right)$, we see that the limit in (1) always exists.

Definition 4.2. We define the harmonic measure $\omega_{K}(z, \cdot)$ on a compact set $K$ with barycenter $z \in K$ as the weak* limit of $\omega_{U_{j}}(z, \cdot)$, chosen as above.

To use this definition for the Dirichlet problem, we must check that the support of $\omega_{K}(z, \cdot)$ lies on the boundary of $K$. Actually, in Section 5, we are able to give more specific information about $\omega_{K}(z, \cdot)$; see Corollary 5.3.

Lemma 4.3. The support of $\omega_{K}(z, \cdot)$ is contained in $\partial K$.

Proof. Let $W$ be a neighborhood of $\partial K$. Let $\left\{U_{j}\right\}$ be a sequence of domains converging to $K$, and take a sequence $z_{j} \in \partial U_{j}$. Then there exists a subsequence $\left\{z_{j_{k}}\right\}$ that must be converging to some $z_{0} \in K$. Because $z_{j} \in \partial U_{j}$, we know $z_{j}$ is not in $K$. Therefore, the limit of $z_{j_{k}}$ cannot be in the interior of $K$. Thus $z_{0}$ is in $\partial K \subset W$. Consequently, there is $j_{0}$ such that $\partial U_{j} \subset W$ for each $j \geq j_{0}$.

Let $x \in \mathbb{R}^{n} \backslash \partial K$, and take $W$ to be a neighborhood of $\partial K$ such that $x \notin \bar{W}$. There is an $r>0$ such that $\overline{B(x, r)} \cap \bar{W}=\varnothing$. Since $\omega_{U_{j}}(z, \cdot)$ has support on $\partial U_{j}$, which is contained in $\bar{W}$ for large $j$, we have $\omega_{U_{j}}(z, B(x, r))=0$. since $b(x, r)$ is open, the portmanteau theorem shows that

$$
\liminf _{j \rightarrow \infty} \omega_{U_{j}}(z, B(x, r)) \geq \omega_{K}(z, B(x, r)) .
$$

Hence, $\omega_{K}(z, B(x, r))=0$, and $x$ is not in the support of $\omega_{K}(z, \cdot)$.

The following theorem brings our study back to the topic of Jensen measures.

Theorem 4.4. The harmonic measure on $K$ is a Jensen measure on $K$. 
Proof. Because $\omega_{K}(z, \cdot)$ is defined as the weak* limit of probability measures, $\omega_{K}(z, \cdot)$ is a probability measure.

Recall that for $z \in K$, we defined $\mathscr{F}_{z}(K)=\cap \mathscr{g}_{z}(U)$, where $K \subset U$. However, it suffices to see that $\mathscr{F}_{z}(K)=\cap \mathscr{F}_{z}\left(U_{j}\right)$, where $\left\{U_{j}\right\}$ is any sequence of domains converging to $K$. We show $\omega_{K}(z, \cdot) \in \mathscr{J}_{z}\left(U_{j}\right)$ for all $j$.

Pick some $j$. Then let $f$ be a continuous subharmonic function on $U_{j}$. Then

$$
f(z) \leq \int_{\partial U_{l}} f(\zeta) d \omega_{U_{l}}(z, \zeta)
$$

for all $l>j$. Then, taking the weak* limit, we have

$$
f(z) \leq \int_{\partial K} f(\zeta) d \omega_{K}(z, \zeta) .
$$

Therefore, $\omega_{K}(z, \cdot)$ satisfies the subaveraging inequality for every continuous subharmonic function on $U_{j}$, and $\omega_{K}(z, \cdot)$ is a probability measure with support contained in $U_{j}$. Thus $\omega_{K}(z, \cdot)$ must be in $\mathscr{g}_{z}^{c}\left(U_{j}\right)$, which is equal to $\mathscr{g}_{z}\left(U_{j}\right)$ by Theorem 2.1. Thus $\omega_{K}(z, \cdot) \in \mathscr{F}_{z}(K)$.

Following [Gamelin 1978, p. 16], a partial ordering on the set of Jensen measures is defined below. The notation $\mathscr{g}(K)$ is used to stand for the union of all Jensen measures on $K$; that is,

$$
\mathscr{g}(K)=\bigcup_{z \in K} \mathscr{g}_{z}(K) .
$$

Definition 4.5. For $\mu, v \in \mathscr{S}(K)$, we say that $\mu \succeq v$ if for every $\phi \in S(K)$ we have $\mu(\phi) \geq v(\phi)$. Furthermore, a Jensen measure $\mu$ is maximal if there is no $v \succeq \mu$ with $v \neq \mu$ where $v \in \mathscr{g}(K)$.

Lemma 4.6. If $\mu \in \mathscr{F}_{z_{1}}(K)$ and $v \in \mathscr{F}_{z_{2}}(K)$ with $z_{1} \neq z_{2}$, then $\mu$ and $v$ are not comparable.

Proof. Recall that the coordinate functions $\pi_{i}$ are harmonic. Because $z_{1} \neq z_{2}$, they must differ in at least one coordinate, say, the $i$-th. Assume without loss of generality that $\pi_{i}\left(z_{1}\right)>\pi_{i}\left(z_{2}\right)$. Then $\mu\left(\pi_{i}\right)>v\left(\pi_{i}\right)$. However, $-\pi_{i}$ is also harmonic, and so $v\left(-\pi_{i}\right)>\mu\left(-\pi_{i}\right)$. Therefore, $\mu$ and $v$ are not comparable, and if $\mu \succeq v$, then they have a common barycenter.

We now demonstrate that the harmonic measure is maximal with respect to this ordering. The maximality of the harmonic measure proved below is the Littlewood subordination principle [Duren 1970, Theorem 1.7] when $K$ is the closed unit ball in the plane.

Theorem 4.7. For all $z \in K$, the measure $\omega_{K}(z, \cdot)$ is maximal in $\mathscr{f}(K)$. 
Proof. By Lemma 4.6, it suffices to show that $\omega_{K}(z, \cdot)$ is maximal in $\mathscr{g}_{z}(K)$ for any $z \in K$.

Pick any $z_{0} \in K$. We show that $\omega_{K}\left(z_{0}, \cdot\right)$ majorizes every measure $\mu \in \mathscr{F}_{z_{0}}(K)$. Consider a decreasing sequence of regular domains $\left\{U_{j}\right\}$ converging to $K$. Take any $\phi \in S^{c}(K)$. By Proposition 3.6, we may find a sequence $\phi_{j} \in S^{c}\left(U_{j}\right)$ increasing to $\phi$. Furthermore, we extend $\phi$ as $\tilde{\phi} \in C_{0}\left(\mathbb{R}^{n}\right)$, while keeping $\tilde{\phi} \geq \phi_{j}$ for all $j$. Define harmonic functions $\Phi_{j}$ on $U_{j}$ by

$$
\Phi_{j}(x)=\int_{\partial U_{j+1}} \phi_{j}(\zeta) d \omega_{U_{j+1}}(x, \zeta) .
$$

Therefore, since $\phi_{j}$ is subharmonic, $\Phi_{j} \geq \phi_{j}$ on $U_{j+1}$, so

$$
\int_{\partial U_{j+1}} \phi_{j}(\zeta) d \omega_{U_{j+1}}\left(z_{0}, \zeta\right)=\Phi_{j}\left(z_{0}\right)=\mu\left(\Phi_{j}\right) \geq \mu\left(\phi_{j}\right)
$$

Because $\tilde{\phi} \geq \phi_{j}$, we have

$$
\int_{\partial U_{j+1}} \tilde{\phi}(\zeta) d \omega_{U_{j+1}}\left(z_{0}, \zeta\right) \geq \mu\left(\phi_{j}\right)
$$

for all $j$. By taking weak* limits, we have that

$$
\lim _{j \rightarrow \infty} \int_{\partial U_{j+1}} \tilde{\phi}(\zeta) d \omega_{U_{j+1}}\left(z_{0}, \zeta\right)=\int_{\partial K} \phi(\zeta) d \omega_{K}\left(z_{0}, \zeta\right)
$$

The Lebesgue monotone convergence theorem gives

$$
\lim _{j \rightarrow \infty} \mu\left(\phi_{j}\right)=\mu(\phi) .
$$

Therefore, by taking the limit by $j$ of (2), we see

$$
\int_{\partial K} \phi(\zeta) d \omega_{K}\left(z_{0}, \zeta\right) \geq \mu(\phi)
$$

We now have $\omega_{K}\left(z_{0}, \cdot\right) \succeq \mu$. If any $v \in \mathscr{F}_{z_{0}}(K)$ has the property $v \succeq \omega_{K}\left(z_{0}, \cdot\right)$, by the antisymmetry property of partial orderings, we have $v=\omega_{K}\left(z_{0}, \cdot\right)$. Thus the measure $\omega_{K}\left(z_{0}, \cdot\right)$ is maximal in $\mathscr{g}_{z_{0}}(K)$.

The maximality of harmonic measures implies that they are trivial at the points $z \in K$ such that $\Phi_{z}(K)=\left\{\delta_{z}\right\}$, which, by Lemma 5.1, are precisely the fine boundary points.

Corollary 4.8. The harmonic measure $\omega_{K}\left(z_{0}, \cdot\right)$ is equal to $\delta_{z_{0}}$ if and only if $\mathscr{g}_{z_{0}}(K)=\left\{\delta_{z_{0}}\right\}$. 
Proof. Suppose $\omega_{K}\left(z_{0}, \cdot\right)=\delta_{z_{0}}$. Consider the function $\rho(z)=\left\|z-z_{0}\right\|^{2} \in S^{c}(K)$. Then for any $\mu \in \mathscr{F}_{z_{0}}$, by the maximality of $\omega_{K}\left(z_{0}, \cdot\right)$, we have

$$
0=\rho\left(z_{0}\right) \leq \mu(\rho) \leq \int_{\partial K} \rho(\zeta) d \omega_{K}\left(z_{0}, \zeta\right)=\rho\left(z_{0}\right)=0 .
$$

Because $\rho(z)>0$ for all $z \neq 0$, and $\mu$ is a probability measure, we see that $\mu=\delta_{z_{0}}$. Thus $\mathscr{F}_{z_{0}}(K)=\left\{\delta_{z_{0}}\right\}$.

For the reverse implication, we have $\omega_{K}\left(z_{0}, \cdot\right) \in \mathscr{F}_{z_{0}}(K)$ from Theorem 4.4.

\section{The boundary}

Gamelin [1978] introduces a version of Choquet theory for cones of functions on compact sets. (Actually, it applies to sets of functions that are slightly more general than the cones we define.)

Following his guidance, we consider a set $\mathscr{R}$ of functions mapping a compact set $K \subset \mathbb{R}^{n}$ to $[-\infty, \infty)$, with the following properties:

(i) $\mathscr{R}$ includes the constant functions;

(ii) if $c \in \mathbb{R}^{+}$and $f \in \mathscr{R}$, then $c f \in \mathscr{R}$;

(iii) if $f, g \in \mathscr{R}$, then $f+g \in \mathscr{R}$; and

(iv) $\mathscr{R}$ separates the points of $K$.

One then considers a set of $\mathscr{R}$-measures for $z \in K$ defined as the set of probability measures $\mu$ on $K$ such that

$$
f(z) \leq \mu(f)
$$

for all $f \in \mathscr{R}$.

Naturally, our model for $\mathscr{R}$ will be $S(K)$. It then follows that when $\mathscr{R}=S(K)$, the $\mathscr{R}$-measures for $z \in K$ are precisely $\mathscr{F}_{z}(K)$. We now state some classic results from [Gamelin 1978] that we need in the following sections.

One can define the Choquet boundary of $K$ with respect to $S(K)$ as

$$
\mathrm{Ch}_{S(K)} K=\left\{z \in K: \mathscr{F}_{z}(K)=\left\{\delta_{z}\right\}\right\} \text {. }
$$

Many nice properties of the Choquet boundary are known. In particular, we need the following characterization; see also, for example, [Bliedtner and Hansen 1986, VI.4.1; Hansen 1985].

Lemma 5.1. The Choquet boundary of $K$ with respect to $S(K)$ is the fine boundary of $K$; that is,

$$
\mathrm{Ch}_{S(K)} K=\partial_{f} K
$$


Proof. Since the fine topology is strictly finer than the Euclidean topology, any point in the interior of $K$ will also be in the fine interior of $K$, and any point of $\mathbb{R}^{n} \backslash K$ can be separated from $K$ by a Euclidean (and therefore fine) open set. Thus the fine boundary of $K$ is contained in $\partial K$. The result follows immediately from [Poletsky 1997, Theorem 3.3] or [Bliedtner and Hansen 1986, Proposition 3.1], which states that $\mathscr{F}_{z}(K)=\left\{\delta_{z}\right\}$ if and only if the complement of $K$ is non-thin at $z$, that is, $z$ is a fine boundary point of $K$.

The set $\partial_{f} K$ is also called the stable boundary of $K$. In fact, Lemma 5.1 shows that $\mathrm{Ch}_{S(K)} K$ is the finely regular boundary of the fine interior of $K$. For details on finely regular boundary points and related concepts, see [Bliedtner and Hansen 1986, VII.5-7; Hansen 1985].

With this association, the result of Brelot [1971, p. 89] about the stable boundary points of $K$ shows that $\mathrm{Ch}_{S(K)} K$ is dense in $\partial K$.

Theorem 5.2. The fine boundary of $K$ (and therefore the Choquet boundary of $K$ with respect to $S(K))$ is dense in the topological boundary of $K$.

In general, the fine boundary is not closed, as Example 6.2 of Section 6 shows. So we cannot claim that it is the support of measures. Also, as Theorem 5.2 shows, the closure of $\mathrm{O}_{k}$ is the boundary of $K$. In particular, it may coincide with $K$ for porous Swiss cheeses [Gamelin 1969, pp. 25-26].

Recall that a measure $\mu \in M(K)$ is concentrated on a set $E$ if for every set $F \subset K \backslash E$, we have $\mu(F)=0$. A probability measure $\mu$ is concentrated on a set $E$ if and only if $\mu(E)=1$. From [Gamelin 1978, p. 19], we know that all maximal measures are concentrated on $\mathrm{Ch}_{S(K)} K=\partial_{f} K$. With this observation, the next corollary immediately follows from Theorem 4.7 (which states that the harmonic measure is maximal).

Corollary 5.3. For every $z$ in $K$, the harmonic measure with barycenter at $z$ is concentrated on $\partial_{f} K$.

\section{The Dirichlet problem on compact sets}

In the classical setting, we know that any continuous function in the boundary of a domain $D \subset \mathbb{R}^{n}$ extends harmonically to $D$ and continuously to $\bar{D}$ if and only if every point of the boundary is regular. For general compact sets in $\mathbb{R}^{n}$, we have:

Theorem 6.1. If $K$ is a compact set in $\mathbb{R}^{n}$, then any function $\phi \in C\left(\partial_{f} K\right)$ extends to a function in $H(K)$ if and only if the set $\partial_{f} K$ is closed. Also, the solution is given by

$$
\Phi(z)=\int_{\partial_{f} K} \phi(\zeta) d \omega_{K}(z, \zeta), \quad z \in K,
$$

and $H(K)$ is isometrically isomorphic to $C\left(\partial_{f} K\right)$. 
From this it also follows that the swept-out point mass at $z$ onto the complement of $K$ is just $\omega_{K}(z, \cdot)$.

Proof. Suppose the set $\partial_{f} K$ is closed. Consider a continuous function $\phi$ on $\partial_{f} K$. Assume that

$$
\Phi(z)=\int_{\partial_{f} K} \phi(\zeta) d \omega_{K}(z, \zeta), \quad z \in K .
$$

Because $\partial_{f} K$ is closed, by Theorem 5.2, we have $\partial_{f} K=\partial K$. Also, because $\omega_{K}(z, \cdot)=\delta_{z}$ for every $z \in \partial_{f} K$, we see that $\Phi=\phi$ on $\partial_{f} K$.

Let $z_{j}$ be a sequence in $K$ converging to $z_{0} \in \partial_{f} K$. Because $z_{0}$ is in $\partial_{f} K=$ $\mathrm{Ch}_{S(K)} K$, we have $\mathscr{F}_{z_{0}}(K)=\left\{\delta_{z_{0}}\right\}$. Because $\mathscr{f}(K)$ is weak* compact [Gamelin 1978, p. 3], any sequence of measures $\mu_{j} \in \mathscr{F}_{z_{j}}(K)$ must converge weak* to $\delta_{z_{0}}$. In particular, $\omega_{U_{j}}\left(z_{j}, \cdot\right)$ is weak* converging to $\delta_{z_{0}}$. Hence, $\Phi\left(z_{j}\right)$ is converging to $\Phi\left(z_{0}\right)=\phi\left(z_{0}\right)$, and $\Phi$ is continuous at the boundary of $K$.

Because $\partial_{f} K$ is closed, $\phi \in C\left(\partial_{f} K\right)=C(\partial K)$. We extend $\phi$ continuously as $\tilde{\phi} \in C_{0}\left(\mathbb{R}^{n}\right)$, and then define the harmonic functions

$$
h_{j}(z)=\int_{\partial U_{j}} \tilde{\phi}(\zeta) d \omega_{U_{j}}(z, \zeta) .
$$

Because $\tilde{\phi}$ is continuous and $\omega_{U_{j}}(z, \cdot)$ converges weak* to $\omega_{K}(z, \cdot)$,

$$
\lim _{j \rightarrow \infty} h_{j}(z)=\lim _{j \rightarrow \infty} \int_{\partial U_{j}} \tilde{\phi}(\zeta) d \omega_{U_{j}}(z, \zeta)=\int_{\partial K} \phi(\zeta) d \omega_{K}(z, \zeta)=\Phi(z) .
$$

Therefore, $\Phi$ is the pointwise limit of a sequence $\left\{h_{j}\right\}$ of functions harmonic in a neighborhood of $K$. Also, we can take the extension $\tilde{\phi}$ of $\phi$ in such a way that the sequence $\left\{h_{j}\right\}$ is uniformly bounded. It now easily follows that $\Phi$ is continuous on the interior of $K$. Indeed, consider a point $z$ in the interior of $K$. Then there exists a ball $B$ centered at $z$ contained in the interior of $K$. The $h_{j}$ are harmonic functions on $B$ converging pointwise to $\Phi$. Therefore, $\Phi$ is continuous on $B$ by the Harnack principle, and so $\Phi$ is continuous on $K$. Thus we have a continuous function $\Phi$ with representation

$$
\Phi(z)=\int_{\partial K} \phi(\zeta) d \omega_{K}(z, \zeta), \quad z \in K .
$$

Since $\Phi$ is continuous on $K$ by [Poletsky 1997], to check that $\Phi \in H(K)$, all that remains is to show that $\Phi$ is averaging with respect to Jensen measures, that is, the equivalence of the external definition of $H(K)$ and the definition by Jensen measures. So we need to see that $\Phi(z)=\mu_{z}(\Phi)$ for every $\mu_{z} \in \mathscr{F}_{z}(K)$ and for every $z \in K$. Because $h_{j}$ is harmonic on $U_{j}$, we have $h_{j}(z)=\mu_{z}\left(h_{j}\right)$. However, by the Lebesgue dominated convergence theorem,

$$
\mu_{z}(\Phi)=\lim _{j \rightarrow \infty} \mu_{z}\left(h_{j}\right)=\lim _{j \rightarrow \infty} h_{j}(z)=\Phi(z) .
$$


Thus $\Phi \in H(K)$.

For the converse, suppose $\partial_{f} K$ is not closed. Then there is a point $z_{0} \in \partial K \backslash \partial_{f} K$. Since $z_{0}$ is not in $\partial_{f} K$, by Corollary $4.8, \omega_{K}\left(z_{0}, \cdot\right)$ is not trivial. Therefore, we can find a set $E \subset \partial K$ such that $\omega_{K}\left(z_{0}, E\right)>0$, with $E$ in the complement of $B\left(z_{0}, r\right)$ for some $r>0$. Consider a continuous function $f$ on $\partial K$ such that $f=1$ on $\partial K$ outside $B\left(z_{0}, r\right)$ and $f=0$ on $B\left(z_{0}, r / 2\right) \cap \partial K$. Then

$$
\int_{\partial K} f(\zeta) d \omega_{K}\left(z_{0}, \zeta\right)>\omega_{K}\left(z_{0}, E\right), \quad z \in K
$$

However, $f\left(z_{0}\right)=0$. Thus there can be no function in $H(K)$ that agrees with $f$ on the boundary of $K$.

Example 6.2. The following is a simple example of a compact set $K \subset \mathbb{R}^{n}, n \geq 3$, in which the fine boundary is not closed. The set $K$ is obtained from the closed unit ball $\bar{B} \subset \mathbb{R}^{n}$ by deleting a sequence $\left\{B\left(z_{n}, r_{n}\right)\right\}_{n=1}^{\infty}$ of open balls whose centers and radii tend to zero. We take the centers to be $z_{n}=\left(2^{-n}, 0, \ldots, 0\right) \in \mathbb{R}^{n}$ and the radii $0<r_{n}<2^{-n-2}$. This is analogous to the "road runner" example of Gamelin [1969, Figure 2] and the Lebesgue spine [2001, p. 187].

By Theorem 6.1, one cannot expect a continuous solution for the Dirichlet problem on an arbitrary compact set, even with continuous boundary data. Therefore, at this point we consider the following broader class of solutions with weaker continuity requirement.

Definition 6.3. Let $f H^{c}(K)$ be the class of finely continuous functions on $K$ that are finely harmonic on the fine interior of $K$ and continuous and bounded on $\partial_{f} K$.

We saw in Definition 3.4 (via Jensen measures) that $H(K)$ consists of the functions in $C(K)$ satisfying the averaging property with respect to $\mathscr{E}(K)$, and by Definition 3.2 (interior) that it can also be seen as the $C(K)$ that are finely harmonic on the fine interior of $K$. Therefore, in the definition of $f H^{c}(K)$, we have maintained the finely harmonic requirement while requiring continuity only on the boundary $\partial_{f} K$ (to match the boundary data). In fact, Theorem 6.5 below shows that the functions in $f H^{c}(K)$ also satisfy the averaging property with respect to $\mathscr{g}(K)$.

Theorem 6.5 shows that the Dirichlet problem on compact sets $K \subset \mathbb{R}^{n}$ is solvable in the class of functions $f H^{c}(K)$ for boundary data that is continuous and bounded on $\partial_{f} K$. The functions that are continuous and bounded on $\partial_{f} K$ are denoted $C_{b}\left(\partial_{f} K\right)$. For this we need the following theorem.

Theorem 6.4 [Fuglede 1972, Theorem 11.9]. The pointwise limit of a pointwise convergent sequence of finely harmonic functions $u_{m}$ in $U$, a finely open subset of $\mathbb{R}^{n}$, is finely harmonic, provided that $\sup _{m}\left|u_{m}\right|$ is finely locally bounded in $U$. 
Theorem 6.5. For every $\phi$ lying in $C_{b}\left(\partial_{f} K\right)$, that is, continuous and bounded on $\partial_{f} K$, there is a unique $h_{\phi} \in f H^{c}(K)$ equal to $\phi$ on $\partial_{f} K$. Moreover, $h_{\phi}$ satisfies the averaging property for $\mathscr{F}(K)$, and in particular,

$$
h_{\phi}(x)=\int_{\partial_{f} K} \phi(\zeta) d \omega_{K}(x, \zeta), \quad x \in K .
$$

Proof. Let $\phi \in C_{b}\left(\partial_{f} K\right)$, and for $x \in \overline{\partial_{f} K}$ define

$$
\tilde{\phi}(x)=\limsup _{y \rightarrow x, y \in \partial_{f} K} \phi(y) .
$$

Since $\phi$ is continuous on $\partial_{f} K$, if $x \in \partial_{f} K$, then $\tilde{\phi}(x)=\phi(x)$. Also, $\tilde{\phi}$ is upper semicontinuous, and thus we may find a decreasing sequence of functions $\left\{\phi_{k}\right\}$ that are continuous on $\overline{\partial_{f} K}$ and converge pointwise to $\tilde{\phi}$. Then we extend the $\phi_{k}$ to $C_{0}\left(\mathbb{R}^{n}\right)$ as $\hat{\phi}_{k}$. By taking $\tilde{\phi}_{k}=\min \left\{\hat{\phi}_{1}, \hat{\phi}_{2}, \ldots, \hat{\phi}_{k}\right\}$, we can make the extensions be decreasing. Consider a decreasing sequence of regular domains $U_{j}$ converging to $K$. Let $u_{j, k}$ be the solution of the Dirichlet problem on $U_{j}$ for $\tilde{\phi}_{k}$. Since the measures $\omega_{U_{j}}(x, \cdot)$ weak* converge to $\omega_{K}(x, \cdot)$, we have that $\lim _{j} u_{j, k}=\int \tilde{\phi}_{k} d \omega_{K}:=u_{k}$. Since the $\tilde{\phi}_{k}$ are decreasing, $u_{k}$ must also be decreasing. Indeed, we let $h_{\phi}=\lim u_{k}$.

Take any $\mu \in \mathscr{g}(K)$. Then $\mu \in \mathscr{F}_{z_{0}}\left(U_{j}\right)$ for all $j$ and some $z_{0} \in K$. Since $u_{j, k}$ is harmonic, we have $\mu\left(u_{j, k}\right)=u_{j, k}\left(z_{0}\right)$. However, by the Lebesgue dominated convergence theorem, we have $\lim _{j} \mu\left(u_{j, k}\right)=\mu\left(u_{k}\right)$, and so $\mu\left(u_{k}\right)=u_{k}\left(z_{0}\right)$. Since the sequence $\left\{u_{k}\right\}$ is decreasing pointwise to $h_{\phi}$, we have $\mu\left(h_{\phi}\right)=h_{\phi}\left(z_{0}\right)$, by the same theorem. Thus $h_{\phi}$ satisfies the averaging property on $\mathscr{f}(K)$. Since $\omega_{K}(z, \cdot)$ lies in $\mathscr{g}(K)$ for all $z \in K$, we see that

$$
h_{\phi}(z)=\int_{\partial_{f} K} h_{\phi}(\zeta) d \omega_{K}(z, \zeta) .
$$

We now show that $h_{\phi}=\phi$ on $\partial_{f} K$. For any $x \in \mathbb{O}_{k}$, we know $\omega_{K}(x, \cdot)=\delta_{x}$, and

$$
u_{k}(x)=\lim _{j \rightarrow \infty} u_{j, k}(x)=\int \tilde{\phi}_{k}(\zeta) d \omega_{K}(x, \zeta)=\tilde{\phi}_{k}(x)
$$

Thus $u_{k}(x)=\tilde{\phi}_{k}(x)$ for all $x \in \partial_{f} K$, and so

$$
h_{\phi}(x)=\lim _{k \rightarrow \infty} u_{k}(x)=\lim _{k \rightarrow \infty} \tilde{\phi}_{k}(x)=\phi(x)
$$

for all $x \in \partial_{f} K$.

To see that $h_{\phi}$ is finely harmonic, we use Theorem 6.4. Observe that $u_{k}$ is the pointwise limit of the harmonic (and therefore finely harmonic) functions $u_{j, k}$, and the solution $h_{\phi}$ is the pointwise limit of $u_{k}$. From the construction of these functions, it is clear that they are bounded.

Corollary 6.6. The set $C_{b}\left(\partial_{f} K\right)$ is isometrically isomorphic to $f H^{c}(K)$. 
Proof. The previous theorem establishes the homomorphism taking $C_{b}\left(\partial_{f} K\right)$ to $f H^{c}(K)$. Observe that $\left.h\right|_{\partial_{f} K} \in C_{b}\left(\partial_{f} K\right)$ for every $h \in f H^{c}(K)$. The uniqueness of the solution shows that $\left.h\right|_{\partial_{f} K}$ extends as $h$. Furthermore, the isometry follows directly from the integral representation in the previous theorem.

\section{Acknowledgements}

It is a pleasure to thank Stephen J. Gardiner, Leonid Kovalev, and Gregory Verchota for stimulating discussions related to the topic of this article. I am especially grateful to Eugene Poletsky for his excellent guidance and support. I would also like to thank the referee for a careful review and for valuable comments that improved this paper.

\section{References}

[Armitage and Gardiner 2001] D. H. Armitage and S. J. Gardiner, Classical potential theory, Springer, London, 2001. MR 2001m:31001 Zbl 0972.31001

[Bliedtner and Hansen 1975] J. Bliedtner and W. Hansen, "Simplicial cones in potential theory", Invent. Math. 29:2 (1975), 83-110. MR 52 \#8470 Zbl 0308.31011

[Bliedtner and Hansen 1978] J. Bliedtner and W. Hansen, "Simplicial cones in potential theory, II: Approximation theorems", Invent. Math. 46:3 (1978), 255-275. MR 58 \#11473 Zbl 0369.31005

[Bliedtner and Hansen 1986] J. Bliedtner and W. Hansen, Potential theory, Springer, Berlin, 1986. MR 88b:31002 Zbl 0706.31001

[Brelot 1971] M. Brelot, On topologies and boundaries in potential theory, Lecture Notes in Mathematics 175, Springer, Berlin, 1971. MR 43 \#7654 Zbl 0222.31014

[Cole and Ransford 1997] B. J. Cole and T. J. Ransford, "Subharmonicity without upper semicontinuity", J. Funct. Anal. 147:2 (1997), 420-442. MR 98h:31005 Zbl 0873.31007

[Cole and Ransford 2001] B. J. Cole and T. J. Ransford, "Jensen measures and harmonic measures", J. Reine Angew. Math. 541 (2001), 29-53. MR 2003c:31002 Zbl 0988.31002

[Debiard and Gaveau 1974] A. Debiard and B. Gaveau, "Potentiel fin et algèbres de fonctions analytiques, I”, J. Functional Analysis 16 (1974), 289-304. MR 52 \#1326 Zbl 0297.31004

[Duren 1970] P. L. Duren, Theory of $H^{p}$ spaces, Pure and Applied Mathematics 38, Academic Press, New York, 1970. MR 42 \#3552 Zbl 0215.20203

[Fuglede 1972] B. Fuglede, Finely harmonic functions, Lecture Notes in Mathematics 289, Springer, Berlin, 1972. MR 56 \#8883 Zbl 0248.31010

[Gamelin 1969] T. W. Gamelin, Uniform algebras, Prentice-Hall, Englewood Cliffs, NJ, 1969. Reprinted Chelsea, New York, 1984. MR 53 \#14137

[Gamelin 1978] T. W. Gamelin, Uniform algebras and Jensen measures, London Mathematical Society Lecture Note Series 32, Cambridge University Press, 1978. MR 81a:46058 Zbl 0418.46042

[Hansen 1985] W. Hansen, "Harmonic and superharmonic functions on compact sets", Illinois J. Math. 29:1 (1985), 103-107. MR 86b:31019 Zbl 0545.31009

[Hervé 1962] R.-M. Hervé, "Recherches axiomatiques sur la théorie des fonctions surharmoniques et du potentiel”, Ann. Inst. Fourier (Grenoble) 12 (1962), 415-571. MR 25 \#3186 Zbl 0101.08103 
[Poletsky 1997] E. A. Poletsky, "Approximation by harmonic functions", Trans. Amer. Math. Soc. 349:11 (1997), 4415-4427. MR 98k:31006 Zbl 0889.32017

Received February 25, 2011. Revised July 8, 2011.

TONY L. PERKINS

DEPARMENT OF MATHEMATICS

215 CARNEGIE BUILDING

SYRACUSE UNIVERSITY

SYRACUSE, NY 13244-1150

UNITED STATES

toperkin@syr.edu 


\title{
PACIFIC JOURNAL OF MATHEMATICS
}

\author{
http://pacificmath.org \\ Founded in 1951 by \\ E. F. Beckenbach (1906-1982) and F. Wolf (1904-1989)
}

\section{EDITORS}

V. S. Varadarajan (Managing Editor)

Department of Mathematics

University of California

Los Angeles, CA 90095-1555

pacific@math.ucla.edu

Vyjayanthi Chari

Department of Mathematics

University of California

Riverside, CA 92521-0135

chari@math.ucr.edu

\section{Robert Finn}

Department of Mathematics Stanford University

Stanford, CA 94305-2125

finn@math.stanford.edu

Kefeng Liu

Department of Mathematics

University of California

Los Angeles, CA 90095-1555

liu@math.ucla.edu
Darren Long

Department of Mathematics

University of California

Santa Barbara, CA 93106-3080

long@math.ucsb.edu

Jiang-Hua Lu

Department of Mathematics

The University of Hong Kong

Pokfulam Rd., Hong Kong jhlu@maths.hku.hk

Alexander Merkurjev

Department of Mathematics

University of California

Los Angeles, CA 90095-1555

merkurev@math.ucla.edu
Sorin Popa

Department of Mathematics University of California

Los Angeles, CA 90095-1555 popa@math.ucla.edu

Jie Qing

Department of Mathematics

University of California

Santa Cruz, CA 95064

qing@cats.ucsc.edu

Jonathan Rogawski

Department of Mathematics

University of California

Los Angeles, CA 90095-1555

jonr@math.ucla.edu

\section{PRODUCTION}

pacific@math.berkeley.edu

\section{SUPPORTING INSTITUTIONS}

ACADEMIA SINICA, TAIPEI

CALIFORNIA INST. OF TECHNOLOGY INST. DE MATEMÁTICA PURA E APLICADA KEIO UNIVERSITY

MATH. SCIENCES RESEARCH INSTITUTE NEW MEXICO STATE UNIV.

OREGON STATE UNIV.

\author{
STANFORD UNIVERSITY \\ UNIV. OF BRITISH COLUMBIA \\ UNIV. OF CALIFORNIA, BERKELEY \\ UNIV. OF CALIFORNIA, DAVIS \\ UNIV. OF CALIFORNIA, LOS ANGELES \\ UNIV. OF CALIFORNIA, RIVERSIDE \\ UNIV. OF CALIFORNIA, SAN DIEGO \\ UNIV. OF CALIF., SANTA BARBARA
}

\author{
UNIV. OF CALIF., SANTA CRUZ \\ UNIV. OF MONTANA \\ UNIV. OF OREGON \\ UNIV. OF SOUTHERN CALIFORNIA \\ UNIV. OF UTAH \\ UNIV. OF WASHINGTON \\ WASHINGTON STATE UNIVERSITY
}

These supporting institutions contribute to the cost of publication of this Journal, but they are not owners or publishers and have no responsibility for its contents or policies.

See inside back cover or pacificmath.org for submission instructions.

The subscription price for 2011 is US \$420/year for the electronic version, and \$485/year for print and electronic.

Subscriptions, requests for back issues from the last three years and changes of subscribers address should be sent to Pacific Journal of Mathematics, P.O. Box 4163, Berkeley, CA 94704-0163, U.S.A. Prior back issues are obtainable from Periodicals Service Company, 11 Main Street, Germantown, NY 12526-5635. The Pacific Journal of Mathematics is indexed by Mathematical Reviews, Zentralblatt MATH, PASCAL CNRS Index, Referativnyi Zhurnal, Current Mathematical Publications and the Science Citation Index.

The Pacific Journal of Mathematics (ISSN 0030-8730) at the University of California, c/o Department of Mathematics, 969 Evans Hall, Berkeley, CA 94720-3840, is published monthly except July and August. Periodical rate postage paid at Berkeley, CA 94704, and additional mailing offices. POSTMASTER: send address changes to Pacific Journal of Mathematics, P.O. Box 4163, Berkeley, CA 94704-0163.

PJM peer review and production are managed by EditFLOW ${ }^{\mathrm{TM}}$ from Mathematical Sciences Publishers.

PUBLISHED BY PACIFIC JOURNAL OF MATHEMATICS

at the University of California, Berkeley 94720-3840

A NON-PROFIT CORPORATION

Typeset in LATEX

Copyright $(2011$ by Pacific Journal of Mathematics 


\section{PACIFIC JOURNAL OF MATHEMATICS}

Volume $254 \quad$ No. $1 \quad$ November 2011

A mean curvature estimate for cylindrically bounded submanifolds

Luis J. AlíAS and MARCos DAJCZER

Weyl group multiple Dirichlet series of type $C$

JENNIFER BEINEKE, BENJAMIN BRUBAKER and SHARON FrECHETTE

Milnor open books of links of some rational surface singularities

MOHAN BHUPAL and BURAK OZBAGCI

Simple closed curves, word length, and nilpotent quotients of free groups

KHALID BOU-RABEE and ASAF HADARI

Strong submodules of almost projective modules

GÁBOR BRAUN and JAN TRLIFAJ

Interlacing log-concavity of the Boros-Moll polynomials

William Y. C. Chen, LARry X. W. WAng and ERnest X. W. Xia

Schwarzian norms and two-point distortion

Martin Chuaqui, Peter Duren, William Ma, Diego Mejía, David

MINDA and BRAD OSGOOD

The principle of stationary phase for the Fourier transform of $D$-modules

JIANGXUE FANG

Monotonicity and uniqueness of a 3D transonic shock solution in a conic nozzle with

variable end pressure

JUN LI, ZHOUPING XIN and HUICHENG YIN

Refined open noncommutative Donaldson-Thomas invariants for small crepant resolutions

KENTARO NAGAO

The Dirichlet problem for harmonic functions on compact sets

TONY L. PERKINS

Extension of an analytic disc and domains in $\mathbb{C}^{2}$ with noncompact automorphism group

\section{MINJU SONG}

Regularity of the first eigenvalue of the $p$-Laplacian and Yamabe invariant along geometric flows

ER-MIN WANG and YU ZHENG 